\title{
CALCHI DI PROVENIENZA ROMANZA NELLO SLOVENO OCCIDENTALE
}

Il calco è il processo linguistico dove, contrariamente al prestito, si imita solo il contenuto semantico dell'espressione in una lingua straniera, o anche in un dialetto della stessa lingua, se si tratta di un calco semantico; oppure dove si imita la struttura in un altro sistema linguistico, e in tal caso abbiamo a che fare con un calco sintattico, strutturale. Bruno Migliorini ha dedicato al problema del calco un succinto studio, dove delimita chiaramente i due processi linguistici: »La forma più elementare di scambio linguistico che consegue a una simbiosi più o meno profonda tra due comunità linguistiche è quella del prestito, cioè l'imitazione più o meno esatta di vocaboli altrui, nella loro forma e nel significato.« Il calco, invece, è per il Migliorini l'imitazione dello spirito informatore, e questo procedimento »implica un più alto livello culturale e un maggior grado di bilinguismo. ${ }^{1}$

L'illustre linguista italiano mette dunque in rilievo due condizioni o almeno due fattori perché si possa ricorrere al calco. La prima, quella di un più alto livello culturale, sembra ovvia per il fatto stesso che il ricorrere al calco esige una certa creatività nella propria lingua oltreché una esatta conoscenza del significato ${ }^{2}$ nella lingua altrui, anche se ci sono casi, particolarmente attraenti per il linguista, di una erronea interpretazione. Il Migliorini cita, per l'area linguistica che ci occupa più da vicino, ${ }^{3}$ l'opinione espressa nel lontano 1933 dall'italianista croato Mirko Deanović ${ }^{4}$ secondo il quale, seguiamo la citazione del Migliorini, «L'influenza dell'italiano si è in complesso esercitata in forma

1 Bruno Migliorini, Calco e irradiazione sinonimica, Boletin del Instituto Caro y Cuervo, IV, 1948. Citiamo da Saggi linguistici, Firenze 1957, pp. 11-22. L'Autore, in questo studio, traccia anche la storia della terminologia.

2 Roberto Gusmani, Per una tipologia del calco linguistico, «Incontri linguistici» 1, Pisa-Trieste (1974). L'autore osserva, ad esempio, che in italiano sheriff è rimasto come prestito, alquanto adattato, sceriffo, dunque non tradotto, perché l'originaria composizione per la sincronia non era più motivata, nemmeno per un inglese; al contrario, ingl. outlaw, perché trasparente, è potuto essere tradotto in italiano con fuorilegge. L'autore ha riunito una serie di preziosi contributi sul prestito e sul calco nella raccolta Saggi sull'interferenza linguistica, 2.da ed., Firenze, 1986, pp. 217-249.

3 Si veda Mitja Skubic, Romanske jezikovne prvine na slovenski zahodni jezikovni meji /Elementi linguistici romanzi nello sloveno occidentale/, Ljubljana, 1997.

4 Mirko Deanović, Osservazioni sulle origini dei calchi linguistici, «Archivum Romanicum», XVIII, 1934, pp. 129-142, e Sulle origini dei calchi linguistici, Atti del III Congresso internazionale dei linguisti, Firenze 1935, pp. 219-226. 
diversa sullo sloveno e sul serbocroato: nella prima lingua predominano i prestiti, nella seconda invece i calchi: e ciò specialmente per il tramite della cultura di Ragusa e delle numerose traduzioni di opere italiane.» ${ }^{5}$

Sarà senza dubbio vero che per la creazione di un calco ci vuole nella lingua ricevente un grado di cultura abbastanza alto. Per il prestito non è necessario nessun particolare sforzo spirituale, giacché un termine straniero si accetta e adatta, per lo più, al sistema morfonologico, mentre il calco esige, oltre ad una buona conoscenza del significato del termine in questione nella lingua straniera, anche la capacità di trovare un'adeguata espressione nella propria. $\mathrm{E}$ da questo punto di vista il Deanović avrà ragione. Sarà però altrettanto vero che il romanista croato, raguseo di nascita, avrà tenuto conto soprattutto della lingua letteraria rinascimentale ragusea, forse in particolar modo delle opere di Marin Držić/Marino D'Arsa, il celebre drammaturgo raguseo (1508 ca. - 1567). Nel suo contributo, il Deanović cita anche dalla lingua colloquiale in Dalmazia dove il prestito di provenienza veneta è abbondantemente presente, anche oggi, e lo era ancora di più nelle epoche passate. Basta richiamarsi ai noti studi dello Schuchardt e del Tagliavini. ${ }^{6}$ Assicura il Deanović che il calco nel parlato è scarso, mentre abbonda nei testi letterari, anche perché nei primi rimatori ragusei è calcato il frasario trovadorico-petrarchesco. Il Deanović, poi, pare non si sia occupato delle interferenze linguistiche italo-slovene ${ }^{7} \mathrm{e}$, per quel che ne so, lo sloveno non lo conosceva. Non si può, di conseguenza, sapere su quale fonte fosse fondata la sua convinzione, ammettendo che sia riportata fedelmente, riguardo allo sloveno. Si può accettare la sua idea di un più alto livello culturale quando si constatano calchi di una certa levatura intellettuale. Va tenuta presente, comunque, la formulazione del Migliorini il quale postula saggiamente, per il calco, un maggior grado di bilinguismo, una simbiosi, diremmo, più stretta. I casi di contatto tra due lingue, e culture, che parlano contro tale convinzione esistono, sì; si spiegano, probabilmente, con il distacco tra le etnie in questione, a prescindere dal grado di cultura di ciascuna di esse. La vera inondazione di arabismi lessicali, quasi esclusivamente sostantivi, nelle lingue ibero-romanze e il più che modesto numero di calchi dall'arabo in spagnolo e in portoghese, malgrado la secolare convivenza, potrebbero esserne una prova.

2. Abbiamo voluto riunire, qui, alcuni casi del calco semantico che crediamo di provenienza romanza, italiana o friulana che sia, nelle parlate occidentali slovene, vale a dire in quelle parti della regione Friuli-Venezia Giulia dove esiste ancora l'etnia slovena. Ci siamo limitati al territorio dove l'etnia slovena da secoli convive con quella

5 La citazione non si trova, tale e quale, in nessuno dei due studi pubblicati dal Deanović e citati dal Migliorini, op. cit., p. 12, né nella discussione sulla relazione del Deanović, alla quale presero parte Rohlfs, Bartoli e Guidi. C'è da supporre che il romanista croato abbia comunicato il suo pensiero al Migliorini in privato, in un'altra occasione.

6 Hugo Schuchardt, Slavo-deutsches und Slavo-italienisches, Graz, 1884. - Carlo Tagliavini, Sugli elementi italiani del croato in Italia e Croazia, Roma, 1942, pp. 377-454.

7 Si veda la bibliografia del Deanović in $»$ Studia romanica et anglica zagrabensia «, rispettivamente $9-10(1960)$ e $29-32(1970-71)$. 
friulana e perciò subisce l'influenza linguistica del friulano. Siccome, poi, si tratta della popolazione che vive in Italia dalla fine della prima guerra mondiale o, per quanto riguarda la fascia orientale della provincia di Udine, addirittura dal 1866, il peso linguistico dell'italiano, soprattutto, e del friulano non deve sorprendere. L'italiano è stato ed è ancora sempre l'unica lingua della scuola nella provincia di Udine (Val Canale, Val di Resia, alte valli del Torre e Natisone). Vale lo stesso per la vecchia generazione nel Goriziano e nel Triestino, quella, cioè, che trascorse gli anni giovanili e frequentò la scuola nel periodo tra le due guerre, nel ventennio fascista. Tutti, poi, dai più giovani ai più vecchi, hanno subìto e subiscono l'influenza linguistica dell'italiano per la sua importanza nella vita pubblica e, in special modo, tramite i potenti mezzi di comunicazione. L'alto numero dei prestiti, sempre adattati, nella lingua parlata della gente slovena della regione Friuli-Venezia Giulia non può sorprendere. Si constata un forte influsso friulano per il passato e il sempre crescente influsso dell'italiano. Per le epoche passate sta a nostra disposizione la preziosissima raccolta di testi, moti, risposte, racconti, ricordi personali degli informatori che raccolse l'insigne linguista polacco Jan Ignacy Baudouin de Courtenay in Val Resia, in Valle del Torre e nelle Valli del Natisone durante i numerosi suoi soggiorni in queste valli a partire dal 1873. Inoltre, il ricco materiale di tradizione popolare, fiabe, racconti, indovinelli e molti scritti che rispecchiano la parlata genuina della gente di queste valli. Questo per dire che non si tratta per niente di un livello linguistico elevato. Chi racconta è gente di condizioni umili, di parlata spontanea, poco o niente influenzata dall'istruzione scolastica.

In base a questo materiale ci pare di poter affermare che, a parte l'alto numero di prestiti lessicali, le parlate slovene occidentali abbondano anche di calchi strutturali e semantici per i quali possiamo senza dubbio alcuno congetturare l'origine romanza. Se per un prestito è abbastanza facile constatare la provenienza friulana o italiana, in base all'immagine fonica, poniamo come esempio miedih, langač, ćarta, stria, zornada, planta che mostrano caratteristiche fonetiche friulane, per il calco la valutazione è piuttosto ardua. Per essere sicuri della provenienza friulana di un calco, si dovrebbe constatare che un fenomeno analogo in italiano è sconosciuto. Più importante, per noi, è ovviamente la semplice constatazione che si tratti di provenienza romanza e qui il criterio scelto potrebbe essere sintetizzato così: un fenomeno linguistico che scopriamo nello sloveno occidentale (parlato, scritto) è con tutta probabilità di provenienza romanza quando non se ne constata uno analogo nello sloveno letterario oppure nei dialetti centrali e lo si trova, ovviamente, in una delle lingue romanze con le quali lo sloveno dell' area occidentale è in contatto.

3. Per la parte sintattica ${ }^{8}$ possiamo dire che i calchi appaiono in tutte le categorie morfologiche. Per il sostantivo troviamo delle influenze nel genere, nel caso, nel 1972, pp. 420-438. 
numero. Troviamo, ad es., il nome della città di genere femminile: Tudi če mi daste vso Napoli, ne ostanem $z$ vam 'Anche se mi regalate tutta Napoli, con voi non rimango', Matajur, mentre lo sloveno in questo caso chiederebbe il maschile. Per il duale ha constatato il Pellegrini che l'uso nello sloveno occidentale risulta piuttosto debole, mentre è robusto assai nei dialetti centrali; secondo noi, non c'è da vedere, comunque, in questo fenomeno l'influenza romanza. Però, si sa che le lingue romanze, non proprio tutte, ma l'italiano e il friulano sì, conoscono il plurale di alcuni sostantivi che designano più persone di sesso diverso, come it. $i$ figli, $i$ fratelli, oppure anche una coppia, come gli zii. Un plurale di tale valore in sloveno non esiste e perciò una frase come Moji noni se spomnijo 'I miei nonni ricordano', scritta dalla nipotina, palesa bene l'interferenza romanza. Nella sintassi del pronome potremmo menzionare la ripresa dell'oggetto per mezzo del pronome personale: Roberta ga poznajo puno judi 'Roberto lo conoscono in molti', Matajur, anche l'uso della forma atona accanto a quella tonica: Mene me je všeč bit dol $v$ dolini 'A me mi piace stare giù nella valle', Matajur. II pronome dimostrativo friulano al sarà il modello per lo sloveno to dei verbi atmosferici: to lampa 'al tarlupe'. 9

Per l'aggettivo va ricordato che in sloveno come attributo di regola precede il sostantivo. Vale a dire che possiamo pensare al calco secondo il modello sintattico romanzo quando in sloveno occidentale lo troviamo dietro il sostantivo: Ta nutre je zavrela den lipi kafe črne ' $\mathrm{Ci}$ ha fatto bollire dentro un buon caffè nero', Matičetov.

Nella sintassi del verbo constatiamo parecchi influssi, come ad es. la forma riflessiva poklekniti se 'inginocchiarsi', che in sloveno non è riflessivo, oppure, al contrario, Spomnite de imamo umreti 'Ricordate che dobbiamo morire', Attems, che in sloveno letterario è riflessivo: spomniti se.

Nel costruire il periodo ipotetico va menzionato l'impiego dell'imperfetto, una rarità, conservatasi solo nel dialetto della Val di Resia, per esprimere l'ipotesi irreale: Ma ći bešajo paršle ti drugi, nemici, ni tešajo ga wstrilit 'Se venivano gli altri, i nemici, lo fucilavano', Steenwijk; oppure, con lo stesso valore dell'irreale, un tempo passato: $\breve{C}$ e želodi so bli valic ku muloni, sada si biu martu'Se le ghiande erano grandi come i meloni, a quest'ora eri morto', Tomasetig.

4. Frequente appare anche il calco semantico: il sostantivo oblasti, ad esempio, ha nello sloveno un solo significato, astratto; quando il sostantivo viene usato per dire 'persone importanti, i dirigenti' come nel passo Predsednik Peterle in druge oblasti 'Il presidente Peterle ed altre autorità', Dom, ci rendiamo conto che questo secondo valore è stato suggerito dall'italiano. Il verbo čuti, čuti se si riferisce solo alla percezione uditiva. Quando lo troviamo come l'espressione di un sentimento, si tratterà di un calco secondo il verbo sentire, sentirsi: Pišem temu, ki je deleč za de se bo ču blizu nas 'Scrivo a chi è lontano, perché si senta vicino a noi', Biasutig. Vale lo stesso per il verbo di percezione sensoriale gledati. Nel senso traslato 'cercare di' sarà un calco semantico dall'italiano o dal friulano: Bili so večpart scalpellini, so poznali kamen in ga znal dielat. Zatuo so gledali podobno dielo 'Erano per lo più scalpellini; conoscevano la 
pietra e la sapevano lavorare. Perciò cercavano (= guardavano a trovare) un lavoro simile', Matajur. Possiamo aggiungere ancora una frase pronunciata da una delle informatrici dello Steenwijk: Ci teniamo molto, za rićet po rozojanskin, da to nan stuji fés ta sércu' '... per dirla alla resiana, che questo ci sta al cuore', dove il verbo sloveno stati ricalca il valore di stare/stâ.

5. Una menzione a parte va dedicata alle locuzioni idiomatiche, sintagmi frequentemente usati in una lingua. Di regola non violano la sintassi; sono interessanti, perché così strutturati non sono conosciuti nei dialetti sloveni centrali o nella lingua letteraria. Offrono un buon esempio le espressioni per l'ora quale misura del tempo; consideriamo, infatti, un calco sul modello romanzo sintagmi quali Ma fiesta na učnela ta na čitiere nu puou 'La mia festa è cominciata alle quattro e mezzo', Vartac. Lo sloveno $o b$ pol petih costruisce il sintagma frazionando l'ora in corso e non aggiungendo i quarti o le mezze ore all'ora già compiuta.

Nelle locuzioni idiomatiche che assumono il valore avverbiale appaiono accanto a una preposizione sostantivi, aggettivi, avverbi. Daremo qualche esempio:

$\mathrm{z}$ močjo
'con forza'

za dobroto

'per carità' par sreč, za nesrečo

'per (s)fortuna'

na velik glas

'a gran voce' dobar, bolši kup

'a bon marciat'

za narobe

'par traviars'

par nogah

'lâ a pit'

na odprtem

'all'aperto'
Pokojnega Kuderja je potrla leta 1933 sila fašistov, ki so z močjo izgnali slovenski jezik iz cerkve 'Il compianto Kuder è stato abbattuto nel 1933 dalla violenza fascista, quando con forza fu bandita la lingua slovena dalla chiesa', Cuffolo, 22-7-1945;

Za dobroto, loži me dol pod ciesto 'Per carità, mettimi giù sotto la strada', Tkol. 1972, 62;

Sem jih potisnil $v$ gosto robidovje, kjer, za srečo, Nemca nista šla vohat 'Li ho spinti /i due prigionieri fuggiaschi inglesi/ nel fitto roveto dietro la casa dove, per fortuna, i due tedeschi non andarono a frugare', Cuffolo, 22-6-1944;

Če za nesrečo bi paršu kaj an glih tah mene s telim uprašanjem 'Se per sfortuna qualcheduno dovesse venire proprio da me con una tale domanda', Dom;

Upite na velich glas 'Gridate a gran voce', Attems, 3, 48;

An požgerješ požirek tuoje pive tisto buj dobar kup 'E tracanni la tua birra, quella a buon mercato', Matajur; Je blo za bolši kup 'Era più a buon prezzo', dal parlato; Sin je biu ku za narobe 'Il frut nol iere che par traviars', Tomasetig, 36;

Marija na mušace an zluodi par nogah 'Maria sull'asino e il diavolo a piedi'. Dom;

15. luja je molú parvo sveto mašo na odprtem pred cerkvijo sv. Duha na Varhu'Il 15 luglio disse la sua prima santa messa all'aperto davanti alla chiesa di S. Spirito a Varch', Tkol. 1992, 80. 
In sloveno, le locuzioni idiomatiche citate non sono in uso, anche se sono per lo più comprensibili. I sintagmi, corrispondenti per il significato, sarebbero: nasilno, iz usmiljenja, na srečo, žal, na ves glas, ${ }^{10}$ poceni, za nikamor, peš, na prostem.

6. Aggiungiamo alcuni sintagmi ai quali partecipa un verbo accanto a un elemento nominale; anche qui non abbiamo a che fare con una struttura sintattica che violi la norma della sintassi slovena, abbiamo tuttavia un costrutto sintattico che non trova un corrispondente semantico:

biti

biti za manj

'essere da meno'

Naši pevci niso bili za manj 'I nostri cantori non erano da meno', Matajur;

biti širokega rokava Barnaški kaplan je biu širokega rokava 'Il cappellano di Bar-

'jéssi di mània

làrgia'

dati nas era di maniche larghe', Tkol. 1976, 56;

dati eno roko

'dâ una man'

delati, storiti

delati + professione

'fare + prof.'

delati kont

fâ cont

storiti festo

delati roge

imeti

imeti spanje

Su dali 'no roko za italijansko besedilo/Hanno collaborato, Blasutig (colofone);

An dila ćaljar 'Fa il calzolaio', Mat. I, 92; Zak k Kristus je marigon dielau 'Poiché Cristo faceva il marangone', Tkol. 1973, 65;

Koi un pride ito, ma sdelat kont, da se trofuje ta pred Iesusan Christusan 'Quando viene qui, deve sapere che si trova davanti a Gesù', Mat. III, 306;

Smo storle fiesto tu taverne 'Abbiamo fatto festa in taverna', Vartac;

Sagra je fijesta, ke na se diela ta pred cierkuo 'La sagra è una festa che si fa davanti alla chiesa' Matajur;

Ob vsaki parložnosti vara moža, mu djela roge 'A ogni occasione tradisce il marito, gli fa le corna', Matajur;

Zutra na će pravet karje, ka naco ka na mela spanje 'domattina racconterà abbondantemente, perché stasera aveva sonno', Mat. I, 1132;

Dopo, ben dopo ni so jenjali, so meli spanje 'Eppoi hanno terminato, avevano sonno', Steenwijk, 19;

videti

videti uro

Samantha je zelo srečna, jih ima štier an vsi na videjo ure jo varvat an kokolat 'Samantha/la neonata/ è molto felice, ha quattro nonni che non vedono l'ora di guardarla e coccolarla', Matajur.

10 Il friulano, poi, conosce la stessa locuzione idiomatica che lo sloveno: a dute vôs. 
Nessuna delle locuzioni idiomatiche citate è conosciuta nello sloveno letterario né nei dialetti sloveni centrali dove si incontrerebbe qualcosa come: biti slabši, biti uvideven, pomagati, biti čevljar, biti tesar, upoštevati/zavedati se, praznovati, nasajati roge, biti zaspan, nestrpno čakati.

7. C'è da menzionare, infine, i sintagmi dove appare il pronome personale atono della terza persona, al singolare o al plurale $j o$, jih che funge, solo sintatticamente, da oggetto diretto. Il fenomeno è conosciuto anche allo sloveno, per lo più con il maschile $g a$, però mai nelle locuzioni idiomatiche che citeremo:

narediti jo

reči jo

misliti jo

kombinavati jih
S' mi ju naredu, Tončič, pa boš videu jutre 'Me l'hai fatta,

Tonino, però, vedrai domani', Tomasetig, 54;

Za jo reč te glih 'Per dirla schietta', Dom;

Takrat jo nisem samuo jest takuo mislu 'In quel tempo non

ero solo io a pensarla così', Matajur;

Ist san an majhen puobič /.../ an jih kombinavam vsih

koluorju 'Sono un ragazzino e ne combino di tutti i colori',

Tkol. 1984, 135.

8. Il nostro materiale ci permette non tanto di opporci alla convinzione, o per lo meno di ridimensionarla, secondo la quale il calco sarebbe soprattutto il privilegio di lingua colta, quanto di poter asserire che il calco non è per niente sconosciuto nella lingua parlata. Sarebbe per lo meno difficile difendere l'idea che una locuzione come ne videti ure, un indubbio calco secondo il modulo italiano non veder l'ora, sia ascrivibile solo al linguaggio colto. Sarà vero che in opere di stampo letterario il calco è più frequente del prestito: gli analisti della lingua biblica, comparando la lingua rispettivamente dell'Itala e della Vulgata, offrono delle prove convincenti in merito. Però, la condizione essenziale perché si ricorra al calco sta in una lunga e costante convivenza di due o più etnie e quindi delle rispettive lingue. Così si giunge, in un territorio etnicamente misto o laddove le etnie vivono in un permanente contatto, non necessariamente a un più o meno alto grado di bilinguismo, ma almeno a una discreta conoscenza della lingua altrui; quella, cioè, che fornirà al parlante significati lessicali nuovi, ancora sconosciuti, oppure schemi, strutture da calcare.

Fonti

Attems Karel Mihael Attems, Slovenske pridige/Omelie slovene/, Trst 1993

Mat. I-III J. Baudouin de Courtenay, Materialien zur südslavischen Dialektologie und Ethnographie, St. Petersburg, 1895, 1904, 1913

Mat. IV Id., Materiali per la dialettologia e l'etnografia slava meridionale/za južnoslovansko dialektologijo in etnografijo, IV, Trieste/Trst, 1988

Cuffolo A. Cuffolo, Moj dnevnik/Il mio diario/, Čedad, 1985

Tomasetig A. Tomasetig, Pravce mojga tat an moje mame/Racconti del mio babbo e della mia mamma/, Speter-Trst, 1981

Blasutig M. Blasutig, Ne samuo spomini/Non solo ricordi, Cividale, 1994

Tkol. Trinkov koledar/Il calendario di msgr. Trinko, annuale/, Špeter, 1959- 
Vartac Vartac/Testi dialettali sloveni/, Špeter, 1994

Matičetov 3 lesičice gotrice 3 comari volpicelle/, a cura di M. Matičetov, Ljubljana, 1987

Steenwijk H. Steenwijk, The Slovene dialect of Resia: San Giorgio, Amsterdam- Atlanta, 1992

Matajur Novi Matajur, (settimanale), Čedad, annate 1985, 1986, 1987

Dom Dom, (settimanale), Čedad, annata 1991.

Povzetek

KALKI ROMANSKEGA IZVORA V ZAHODNI SLOVENŠČINI

Prispevek tehta trditev, da je pomenski ali skladenjski kalk zadeva knjižnega jezika, prevzemanje izrazov iz tujega jezika pa stvar govorjenega, torej predvsem neposrednega stika dveh jezikov. Po mnenju hrvaškega italianista Mirka Deanovića, kar sprejema tudi italijanski jezikoslovec Bruno Migliorini, naj bi bil italijanski vpliv na (srbo)hrvaški jezik viden zlasti v dubrovniški renesančni književnosti, v slovenščini pa naj bi bile zaznavne predvsem prevzete besede, posledica neposrednega stika. Naše gradivo, od jezika v pridigah prvega goriškega nadškofa Attemsa in anket poljskega jezikoslovca Jana I. Baudouina de Courtenaya pa preko bogatega ljudskega izročila vse do zapisov naših dni, pa dokaj jasno priča, da so ob številnih prevzetih besedah romanskega, torej furlanskega in italijanskega izvora na zahodni slovenski jezikovni meji obilno zastopani tudi kalki. 